\title{
Development of a Web Based Image Annotation Tool for Lung Immunofluorescent Confocal Images
}

\author{
Shu ISAKA *, Hiroharu KAWANAKA *, V. B. Surya PRASATH **, Bruce J. ARONOW ** and \\ Shinji TSURUOKA* \\ * Division of Electrical and Electronic Engineering, Graduate School of Engineering, Mie University, Mie, Japan \\ ** Division of Biomedical Informatics, Cincinnati Children's Hospital Medical Center, Cincinnati OH 45299 USA \\ ** Department of Biomedical Informatics, University of Cincinnati, Cincinnati OH USA
}

\begin{abstract}
A molecular atlas of the human lung is important to inform basic mechanisms and treatments for lung diseases, and imaging data provide us the foundation upon which to build the lung atlas. For analyzing immunofluorescent confocal images, annotations describing precise anatomical structures are necessary. However, it is hard to annotate increasing images manually. Thus, this study aims to develop an automatic annotation system as a combination of automatic region detection and automatic structure classification modules. As an important and first step to achieving the aim, we developed an efficient annotation data collection tool that will be used collected data to develop the automatic annotation system for the lung atlas. We describe the details of our web based annotation tool that is web based and includes user control.
\end{abstract}

Keywords: Image Annotation, Biomedical Informatics, Lung Image

\section{INTRODUCTION}

A molecular atlas of the human lung is a critical platform for the future research to inform basic mechanisms and treatments for lung diseases. The LungMAP consortium aims to build a mouse/human lung atlas and an integrated database [1]. Currently, many research projects on RNA sequences, proteomics, and imaging [1]-[4] have been reported. In particular, imaging data provides us the foundation to build the lung atlas. Therefore, it is required to understand these visual data and identify a specific region in the images by annotation [1], [5].

The team of LungMAP has already developed the interactive web application, which identified the structures of the given area automatically [1]. This system, however, required the manual input for identification. Moreover, plenty of lung imaging data archived in the database will be extended for the 3D images in the future. It will be so hard for annotators to annotate all of them manually, and the demand for automatic annotation system has been growing.

The authors aim to develop an automatic annotation system for lung images using machine learning techniques. The annotation system will be developed by the combination of (i) Automatic Detection of Region of Interest and (ii) Structure Classification. As the first step of this study, we developed a web-based tool to collect

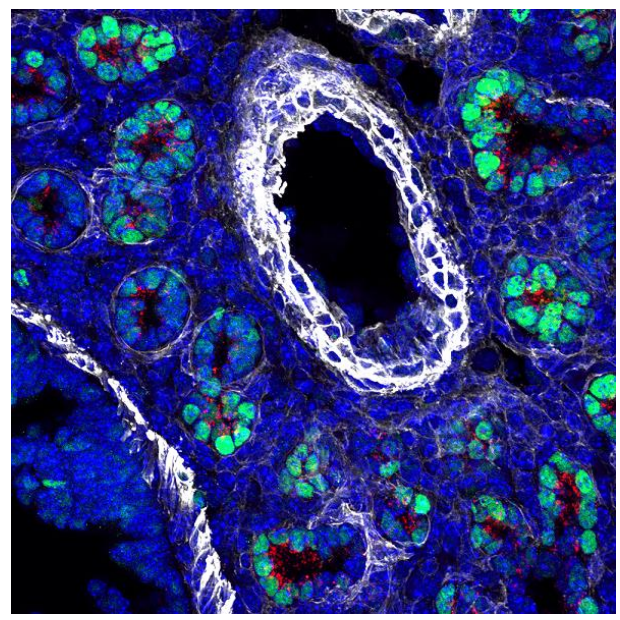

Figure 1: Example of a Lung Immunofluorescent (IF) Confocal Image. Three types of proteins were stained as red, green, and white. In this example, red pixels correspond to protein Sftpc. green, and white pixels mean protein Sox 9 and Acta2, respectively.

annotated lung immunofluorescent images to realize this system. By using the developed system, each region of interest in the collected image will be linked to anatomical structure information, and biomedical informaticians would be able to use them for analyses, identification of tissues and so on. It is one of the benefits of our system.

\section{MATERIALS}

In this study, we focus on immunofluorescent (IF) confocal images of mouse and human lung. The images 


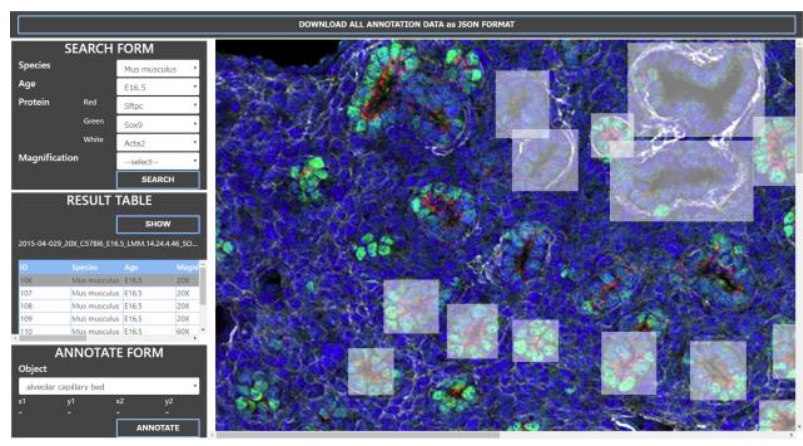

Figure 2: Screenshot of the developed web based annotation tool. System users can annotate with mouse control and all of the annotated data will be stored to the database. White rectangle means ROIs for annotation.

were collected from LungMAP website [6]. In the case of mouse, the IF images were collected from Mus musculus, whose age was from embryo 16.5 days to postnatal 28 days. Human means Homo sapiens and these ages were from 9 months old to 4 years old. All of the images were stained as red, green and white like Figure 1. The meaning of stained colors, i.e. set of stained proteins, depends on each image.

\section{DEVELOPMENT OF DATA COLLECTION TOOL}

A data collection tool is necessary to develop the automatic annotation system with machine learning techniques because machine learning methods require plenty of data to learn the features for classification. Therefore, the authors have developed a data collection and manual annotation tool on the web.

\subsection{System Requirements}

The developed data collection tool should be designed and developed considering the following three points.

1) Architecture for Ubiquitous System

The given lung images are usually provided by pathologists, and they will also annotate the given data. The annotated images are archived in the database for subsequent analyses. Also, most pathologists in the hospital are busy with daily works. Thus, the developed system should be ubiquitous; this means the developed system should not be subjected to any constraints on time and place. For these requirements, a web-based tool is one of the practical approaches because the web-based tool will be easy to ask specialists and able to connect the database with the tool.

2) Filtering of IF Images Based on its Information

The archived IF images have various information such as imaging condition, developmental stage (i.e. age) of the
Table 1: Server Environment

\begin{tabular}{|c|c|}
\hline Machine & Dell PowerEdge T320 \\
\hline OS & Ubuntu 16.0.4 (64bit) \\
\hline CPU & Intel(R) Xeon(R) CPU E5-1410 0 @ 2.80GHz \\
\hline Core & 24 GB \\
\hline RAM & Apache 2.4.18 + Tomcat 8.0.32 \\
\hline Web Server & MySQL 5.7.19-Oubuntu0.16.04.1 \\
\hline Database &
\end{tabular}

subjects, and the combination of antibodies for staining the lung tissue. This information is usually used to search the desired IF images for annotation. Thus, the developed system should have a function to search the images with specific conditions. This function will make the annotators possible to access their desired image(s) easily. 3) Data Structure for Analyses

In addition to the above information, each archived IF image has an experimental condition. Furthermore, the annotated image has many ROIs. In other words, the relationship between the annotated images and annotations is "many to many relationships." In this system, JSON was used to express the collected annotation data.

4) Tissue Extraction from ROI for Annotation

In the developed system, the desired tissue should be extracted with polygons because we cannot express the shape of ROI with a rectangle only. For instance, the shapes of some target tissues are like a circle, and then the ROI may or may not have surplus (or not enough) regions. Thus, automatic segmentation method of desired tissues is required for the developed system.

\subsection{Outline of Developed System}

The authors have developed a web-based tool for annotation of IF images. To develop the system, we used Java with a web framework "Spring Boot", Apache 2.4.18 on Ubuntu 16.04 Server and Tomcat8.0.32. Table 1 shows specification of our server.

Figure 2 shows the screenshot of the developed tool. In the system, the screen consists of four parts; Search Form, Result Table, Annotate Form and Picturebox Area. After system users input (or select) conditions on the search form, and then this web tool connects to the database and shows search results in the result table. After this, we can select the desired IF image by clicking each row.

Further, system users can download all of the annotation data on the web tool, the format of the annotation data is a JSON file. If users develop a parser of the JSON File, they can import the annotated data to their computer and can do advanced analyses. Figure 3 shows 


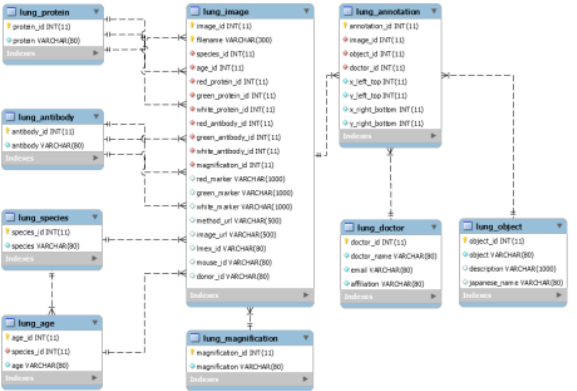

Figure 3: Entity Relationship Diagram. Each image file is related to its experimental condition (such as imaging condition, the set of antibodies, stained proteins, developmental stage of the subject), and multiple annotations will be linked to each image.

the entity-relationship diagram of the database.

\subsection{Actual Use of Developed System and Data Stream}

Figure 4 is the data stream among the user, client, server, and database.

1) Architecture for Ubiquitous System

The desired IF image can be extracted from the database by using specific search conditions. The flow of this process is the following steps (Steps from \#1 to \#9 in Figure 4).

1. (User) Input Filtering Condition

2. (Client) Send Filtering Condition to Server

3. (Server) Search Images from Database

4. (Client) Obtain Filtered Image List

5. (Client) Show Filtered Image List

6. (User) Select Image from the List

7. (Client) Send Image Information

8. (Client) Obtain Image

9. (Client) Show Selected Image

2) Annotating Images

After this, specialists can annotate the images by using a mouse, and all of the annotated data will be stored in the database. The flow of this process is the following steps (Steps from \#10 to \#12 in Figure 4).

10. (User) Annotate Image

11. (Client) Send the Annotation Data to Server

12. (Server) Store Annotation Data to Database

The annotation data can be the both of rectangles and polygons. Figure 5 shows an example of selected ROI with polygons and rectangles.

3) Downloading Annotation data

Users can download (and get) all of the annotation data on the tool, the format of the annotation data is downloaded as a JSON file. If users develop a parser of the JSON File, they can import the annotated data to their computer and can do advanced analyses.

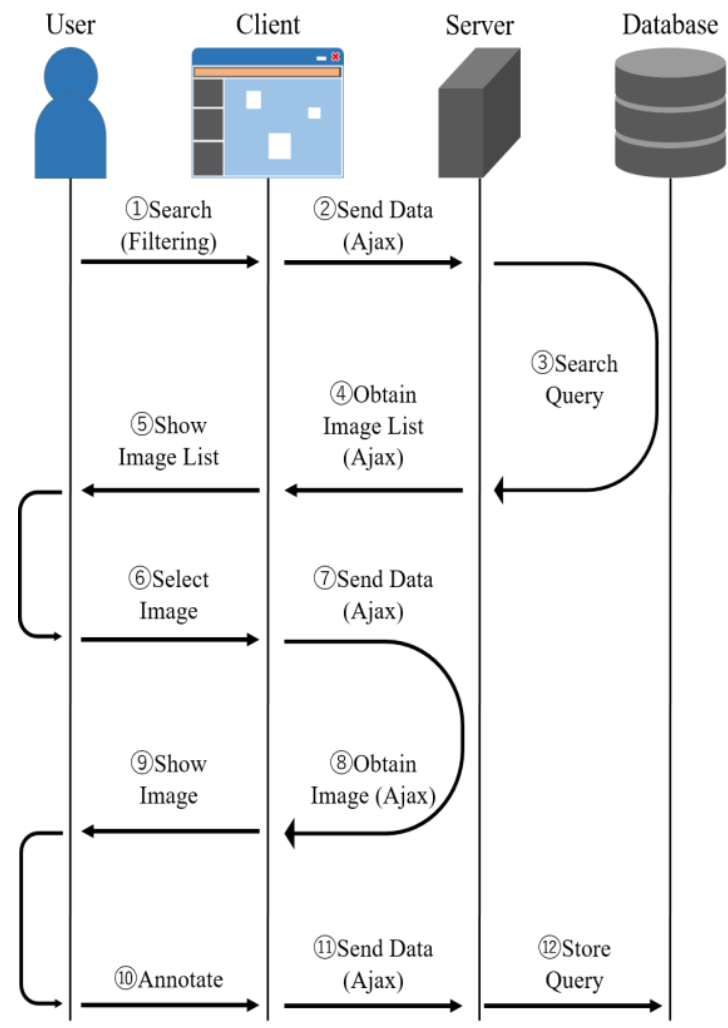

Figure 4: Data flow of developed tool among the users, client, server, and database. (1) Users can filter the images with drop-down lists of search criteria, and select one image from the given list (from \#1 to \#9). (2) Users can Annotate with simple mouse control (from \#10 to \#12).

\section{TOWARD THE FUTURE}

At the moment, the developed system is just a tool for data collection, that means it has no intelligent mechanism for automatic annotation and classification. For instance, system users have to put ROI(s) manually so far. Also, the tissue area should be extracted, recognized appropriately by image recognition techniques. To reach the final goal of this research project, we have to discuss the following methods as future works.

\subsection{ROI Detection and Segmentation}

The IF image has many target tissues for annotation and drawing ROIs on the image is a lot of burden to end users. Also, we have to extract appropriate tissue area from the ROI for accurate annotation. To realize this, we need a technique to detect ROI and get the desired tissue from ROI automatically and accurately. The ROI detection system scans the given image based on the concept of multi-resolution analysis, and then candidates of ROI are determined by using color, shape information. We are now discussing a method for this problem.

Another task is to extract the desired tissue from the 


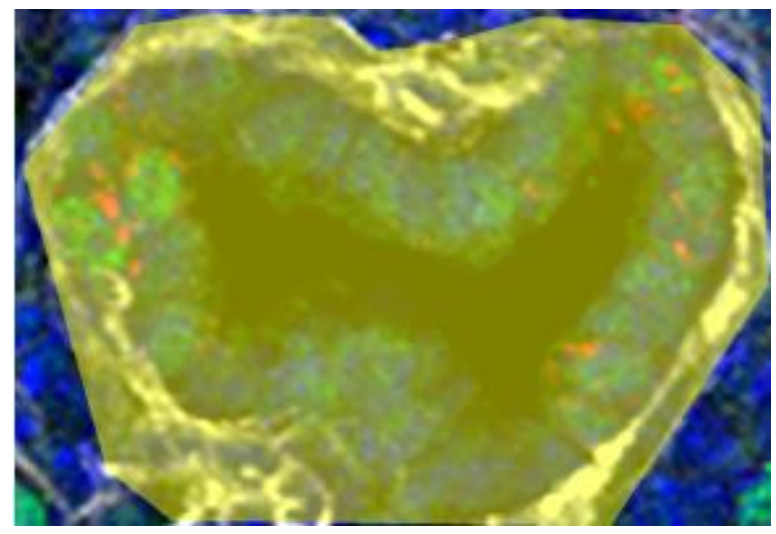

Figure 5: Polygon Annotation. Users can edit this polygon with simple mouse control.

ROI. As mentioned before, there are surplus regions for automatic annotation. We have to remove such area from the ROI for classification. Previously, many segmentation methods are proposed and discussed for practical use. However, the characteristics of medical images are different from others, and this makes the segmentation problem difficult. Figure 6 is an example of segmentation for distal acinar tubule bud of embryo 16.5 days mouse lung. To get this figure, we utilized the GrabCut algorithm [7], GrabCut algorithm is one of the segmentation methods, and its performance is high for general images. In the case of this particular example, the segmentation result looks good, but the obtained result has still surplus regions. It is difficult to apply such segmentation techniques to the given image directly, and some additional concept/techniques will be required [8].

\subsection{Structure Recognition}

The next task will be to develop a method to identify types of the extracted tissues automatically. We will use some methods for image recognition. As the first step, we will discuss features of the extracted tissues and a method to extract them from the tissue images to construct a classifier model $[9,10]$. Further, we will not only use statistical approaches but also state of the art machine learning techniques like Convolutional Neural Networks (CNNs) for highly accurate structure recognition $[11,12,13]$. These are important future work of this study.

\section{CONCLUSION}

A molecular atlas of the human lung is important to know the lung development mechanism, and it is useful for the future medicine and future research. The LungMAP consortium that works for integrated research of gene, protein, anatomy and imaging data to make molecular atlas.

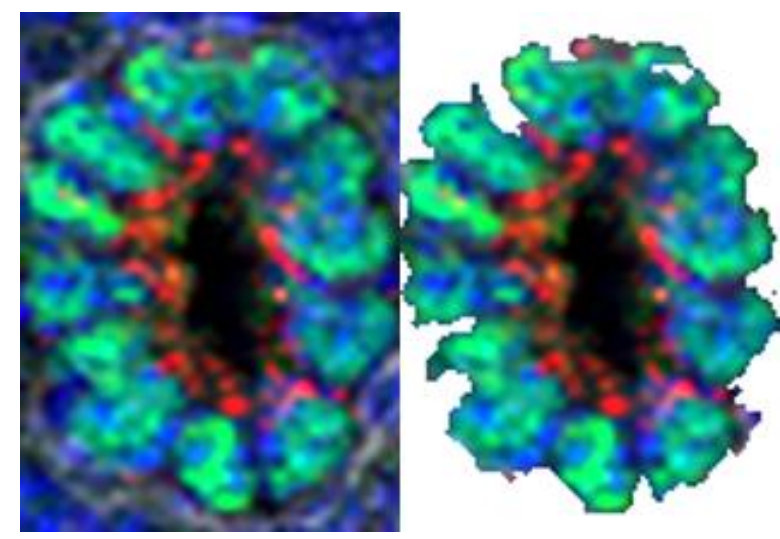

Figure 6: Segmentation Result Example. Distal Acinar Tubule Bud of Embryo 16.5 Days Mouse Lung. Left image is original and right image is the segmentation result.

This study aims to annotate the lung immunofluorescent confocal images automatically. As the first step of this study, the authors developed the manual annotation tool on the web to collect the manual annotation data. In this system, users can filter images, annotate images with both of rectangle and polygon regions easily. This paper also mentioned the current progress and remained problems in this study.

In the future, we will develop an automatic ROI detection and tissue extraction method, and also develop a tissue recognition system. And finally, these systems will be combined for effective automatic annotation for IF images.

\section{REFFERENCES}

1. M. E. Ardini-Poleske et al.; LungMAP: The molecular atlas of lung development program, Am J Physiol Lung Cell Mol Physiol, 313, L733L740, 2017.

2. M. Guo et al.; SINCERA: A Pipeline for single-cell RNASeq profiling analysis, PLoS Comput Biol, 11(11), e1004575, doi:10.1371/journal.pcbi.1004575, 2015.

3. Y. $\mathrm{Xu}$ et al.; Single-cell RNA sequencing identifies diverse roles of epithelial cells in idiopathic pulmonary fibrosis, JCI Insight, 1(20), e90558, doi:10.1172/jci.insight.90558, 2016.

4. G. Clair et al.; Spatially-resolved proteomics: Rapid quantitative analysis of laser capture microdissected alveolar tissue samples, Sci. Rep. 6, 39223, doi: 10.1038/srep39223, 2016.

5. L. Barbe et al.; Toward a confocal subcellular atlas of the human proteome, Molecular \& Cellular Proteomics, 7(3), 499-508, 2007.

6. LungMAP official web site, https://lungmap.net, [Accessed 24 April 2018]. 
7. C. Rother et al.; GrabCut - Interactive Foreground Extraction using Iterated Graph Cuts, ACM Transactions on Graphics (TOG), 23(4), 309-314, 2004.

8. V. B. S. Prasath et al.; Cell nuclei segmentation in glioma histopathology images with color decomposition based active contours, IEEE International Conference on Bioinformatics and Biomedicine (BIBM), 1734-1736, 2015.

9. Fukuma et al.; Feature extraction and disease stage classification for glioma histopathology images, IEEE International Conference on E-health Networking, Application \& Services (HealthCom), 2015.

10. Fukuma et al.; A study on feature extraction and disease stage classification for Glioma pathology images, IEEE International Conference on Fuzzy Systems (FUZZ-IEEE), 2150-2156, 2016.

11. A. Yonekura et al.; Improving the generalization of disease stage classification with deep CNN for glioma histopathological images, IEEE International Conference on Bioinformatics and Biomedicine (BIBM), 1222-1226, 2017.

12. A. Yonekura et al.; Glioblastoma multiforme tissue histopathology images based disease stage classification with deep $\mathrm{CNN}$, International Conference on Informatics, Electronics \& Vision (ICIEV), 2017.

13. A. Yonekura et al.; Automatic disease stage classification of glioblastoma multiforme histopathological images using deep convolutional neural network. Biomedical Engineering Letters, 2018. 